\title{
Conversion of Scattering Parameters to Time-Domain for Imaging Applications: Rules and Examples
}

\author{
Marcelo Bender Perotoni, Claudio José Bordin Jr., and Kenedy Marconi G. dos Santos.
}

\begin{abstract}
This article discusses the conversion of scattering parameters from an antenna input port to the time domain for imaging applications. Frequency-domain measurements offer advantages to time-domain methods: the complex data encoded in the scattering matrix convey target geometric and material information, which can later be used to reproduce the scene image illuminated by the antenna. We discuss three different examples using the inverse discrete Fourier transform. Tradeoffs in the data acquisition, bandwidth, sampling, and topics related to the antennas used as probes are covered.
\end{abstract}

Index Terms-Imaging; Scattering Parameters; Signal Processing for Electromagnetics.

\section{INTRODUCTION}

$\mathbf{C}$ OMPLEX scattering S-parameters are measured in Vector Network Analyzers (VNAs) and provide an understanding of the behavior and performance of linear passive and active elements and systems. Besides real-world measurements, electromagnetic field solvers make wide use of S-parameters as means to evaluate virtual radiofrequency and electromagnetic compatibility (EMC) problems. Though provided as a complex-valued vector uniformly spread across a frequency range, they encode useful information when analyzed in time-domain, in applications such as TDR (timedomain reflectometry), radar [1] [2], near and far-field imaging [3] applied in areas such as breast cancer detection [4], ground-penetrating radar [5] and even non-destructive concrete characterization [6].

The use of transformed frequency-domain data instead of a direct time-domain measurement offers some advantages, such as higher SNR (Signal-to-Noise Ratio) due to a narrowband measurement, which reduces associated the thermal noise, reduction of zero-level drift problems, and the use of known standards, such as the calibration sets of VNAs [7] [8]. For imaging purposes, UWB (Ultra-Wide Band) techniques offer some advantages given the benefit of generating narrow pulses: with the assigned band in the range of $3.1 \mathrm{GHz}$ to 10.6 $\mathrm{GHz}$, a theoretical $0.13 \mathrm{~ns}$ pulse is possible. The analysis of transformed frequency-domain UWB S-parameter data can be performed after the VNA measurement, given the $10 \mathrm{GHz}$ range oscilloscopes availability. However, systems based on

M. B. Perotoni and C. J. Bordin Jr. are with Universidade Federal do ABC (UFABC), Santo André, SP, Brazil (email: marcelo.perotoni,claudio.bordin@ufabc.edu.br).

K. M. G. dos Santos is with Instituto Federal da Bahia (IFBA), Vitória da Conquista, email: kenedymarconi@ifba.edu.br.

Digital Object Identifier: 10.14209/jcis.2021.6. time-domain benefit from faster scanner times and more costeffective devices, particularly with the availability of real-time low GHz range oscilloscopes [3] [9]. Despite FFT/IFFT routines (Fast Fourier Transform / Inverse Fast Fourier Transform) and Chirp-Z Transforms [10] [8] being available in network analyzers and field solvers alike, sometimes a deeper understanding is needed to implement own codes and better design systems that make use of time-domain signals transformed from frequency-domain data sets. Processing resources such as zero-padding, oversampling, filtering, and windowing might be used in addition to the IFFT, which might not be quite clear to the instrument or field solver user.

Previous studies have shown similar applications without delving into the conversion itself [11], [12], [13]. Several techniques dealing with microwave imaging use the transformed frequency-domain data as input to spatial variables, cases of ISAR (Inverse Synthetic Aperture Radar) for farfield identification of targets, typically aircraft [1], throughwall imaging, with algorithms such as Delay and Sum (DS) or Range Migration (RM) [14], or by converting to time domain where indirect information on target positions and respective materials are encoded, case of algorithms such as Single Probe Imaging through Detection and Reconstruction (SPIDR) [15].

This article describes the steps needed to convert complexvalued uniformly sampled S-parameters in the frequency domain to real-valued time-domain responses, providing the necessary steps and mathematical background, thereby covering a missing spot on the existing textbooks and related literature. Specifically, we elaborate further on [16], presenting detailed derivations and illustrative examples. It uses computer simulation and analytical expressions as the S-Parameter or reflection coefficient to analyze different scenarios where the conversion is performed. The text in the sequel is organized as follows: Section II details the computation of the IFFT of scattering parameters. Sections III and IV describe examples of use of the proposed technique in distinct applications. Finally, the conclusions are drawn in Section IV.

\section{COMPUTING THE INVERSE FOURIER TRANSFORM OF A SCATTERING PARAMETER VECTOR}

A band-limited signal $H_{p b}(f)$ can be represented as a product of a function with unlimited support $H(f)$ and a rectangular (rect) function, defined as one in the band $\Delta F=$ $f_{\max }-f_{\min }$ and zero elsewhere [17]: 


$$
H_{p b}(f)=H(f) \cdot \operatorname{rect}\left(\frac{f-f_{o}}{\Delta F}\right),
$$

where the center frequency is defined as

$$
f_{0}=\frac{f_{\max }+f_{\min }}{2}
$$

The product in the frequency domain is equivalent to a convolution in the time domain. If $h(t)$ and $h_{p b}(t)$ are respectively the inverse transforms of $H(f)$ and $H_{p b}(f)$, the convolution can be written as:

$$
h_{p b}(t)=h(t) * \Delta F \cdot \operatorname{sinc}(\Delta F \cdot t) e^{j 2 \pi f_{0} t},
$$

with

$$
\operatorname{sinc}(t)=\frac{\sin (\pi t)}{\pi t} .
$$

The measured samples of (1) can be expressed as the multiplication of (1) by a train of Dirac deltas spaced by $f_{s}$ (comb function) representing the sampling rate of the original data in the frequency domain:

$$
H_{p b, \text { sampled }}(f)=H_{p b}(f) \cdot \sum_{k=-\infty}^{\infty} \delta\left(f-k f_{s}\right) .
$$

The effect of sampling in time domain is felt by way of replicating the pulse at time intervals defined as:

$$
T_{s}=1 / f_{s}
$$

Fig. 1 depicts the process. Due to the convolution with a modulated sinc function, ringing appears in the response. Ideally, the original function $H(f)$ should have low energy above $f_{\max }$ as to reduce the ringing [17]. The sampling effect in the time domain results in the repetition of the pulse at every $1 / f_{s}$ interval. Due to the Nyquist limit, the distance between samples in the frequency domain vector $f_{s}$ should be such that $1 / f_{s}$ is larger than the expected response duration (Fig. 1 ), otherwise aliasing will show up, distorting the computed signal.

Assuming that the time signal is real-valued, its Fourier transform has to be Hermitian [18] - i.e. the real part of the complex data is even and the imaginary part is odd. However, the extracted data from the measurement or simulation is only defined for positive frequencies. Therefore, data at negative frequencies must be defined as conjugate of data at positive frequencies, in an operation so-called "Hermitian Processing" [16]. After Hermitian Processing (denoted $H e\{\}$ ), one obtains:

$H e\left\{H_{p b}(f)\right\}=H(f) *\left[\operatorname{rect}\left(\frac{f-f_{0}}{\Delta F}\right)+\operatorname{rect}\left(\frac{f+f_{0}}{\Delta F}\right)\right]$.

Using the Euler relation, the inverse Fourier transform results in:

$$
\mathcal{F}^{-1}\left(H e\left\{H_{p b}(f)\right\}\right)=h(t) \cdot \Delta F \cdot \operatorname{sinc}(t \Delta F) \cdot \cos \left(2 \pi t f_{0}\right) .
$$
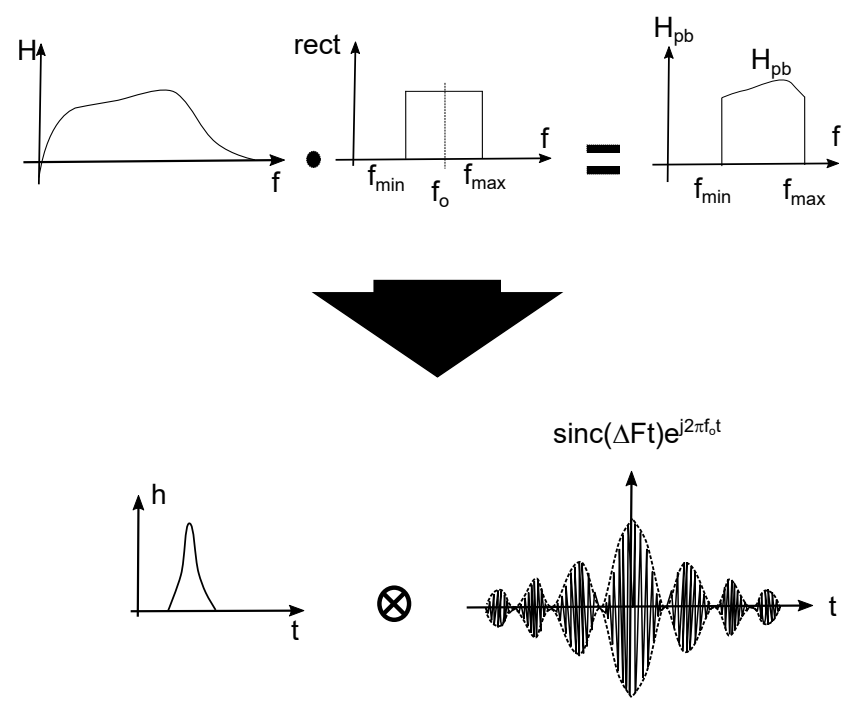

Sampling:
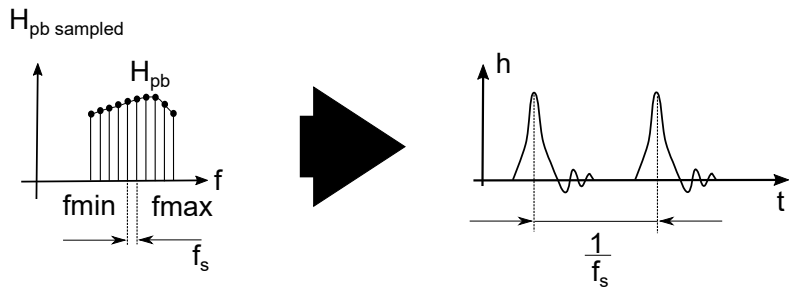

Fig. 1. Effect of truncating the ideal response to a band-pass and the subsequent sampling in time domain. The black arrows show the result of an Inverse Fourier Transform operation.

The original data acquired from either an instrument or from a simulation contains $N$ samples uniformly distributed between $f_{\min }$ and $f_{\max }$, according to Fig. 2. The imported data then is submitted to Hermitian Processing [16] and zeros are inserted between $-f_{\min }$ and $f_{\min }$ with the same sampling step as the original data.

After this procedure, the IFFT of the resulting vector can be computed to determine the respective time-domain pulse, whose time scale (Fig. 2) is determined by the resolution $\Delta t$ and maximum time-span $T_{\max }$, defined as:

$$
\begin{gathered}
\Delta t=\frac{1}{L \cdot\left(f_{i+1}-f_{i}\right)}=\frac{1}{L \cdot f_{s}}, \\
T_{\max }=\frac{1}{f_{i+1}-f_{i}}=\frac{1}{f_{s}},
\end{gathered}
$$

where $\mathrm{L}$ is the total number of frequency samples (including eventual zero-padding), comprising complex data spread between $-f_{\max }$ and $f_{\max } . f_{i}$ and $f_{i+1}$ are consecutive measured data samples in the frequency domain, whose distance is represented by the variable $f_{s}$. Fig. 3 illustrates the relation between both domains. Usually the samples are homogeneous either in simulators or measurement instruments; in case they are not some resampling routine should be applied. Due to zero-padding, $L>N$, unless the measurements starts at DC (zero frequency). 


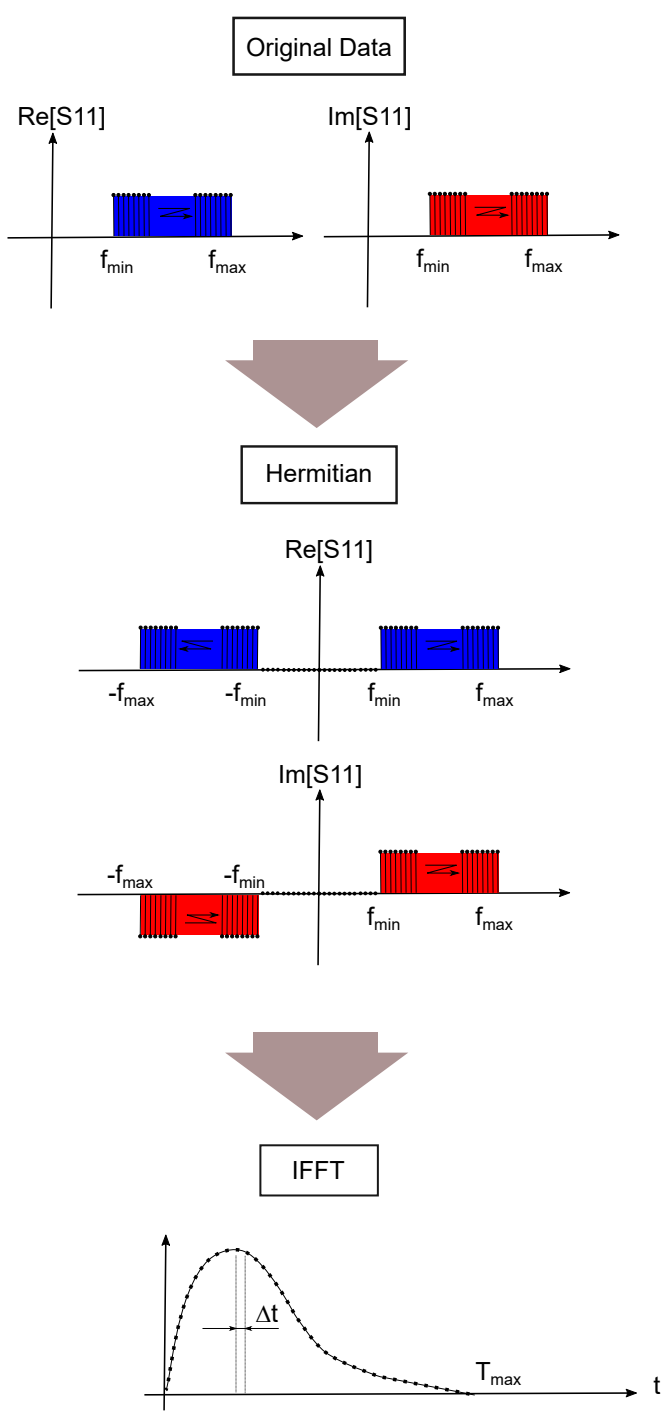

Fig. 2. Hermitian Reconstruction of the original data and its IFFT.

In order to obtain a finer resolution in the time domain, able to resolve nearby details in imaging applications, a large number of samples $N$ and a high maximum frequency $f_{\max }$ are needed. Therefore, this resolution $(\Delta t)$ is determined by the available hardware (VNA, antennas frequency range and associated elements). In case the number of frequency samples $N$ is not large enough, resampling can be applied, as long as the original data is smooth and occasional resonances and peaks are not lost.

Next, practical applications of the afore-described method are presented.

\section{EXAMPLE I - BAND-PASS UNITARY AMPLITUDE FUNCTION}

A unitary amplitude, real function with $N=100$ samples, between $f_{\min }=4 \mathrm{GHz}$ and $f_{\max }=10 \mathrm{GHz}$ is submitted to Hermitian Processing (Section II) leading to the results shown in Fig. 4. After the zero-padding a total number of samples $L=331$ is generated.

The sample distance in time domain is $\Delta t=1 /(331 *$ $60.6 \mathrm{MHz})=0.05 \mathrm{~ns}$, where $60.66 \mathrm{MHz}$ corresponds to the

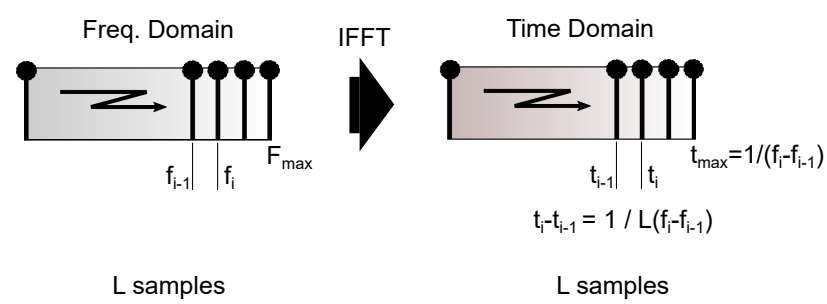

Fig. 3. Relation between the time and frequency scales after the IFFT operation.

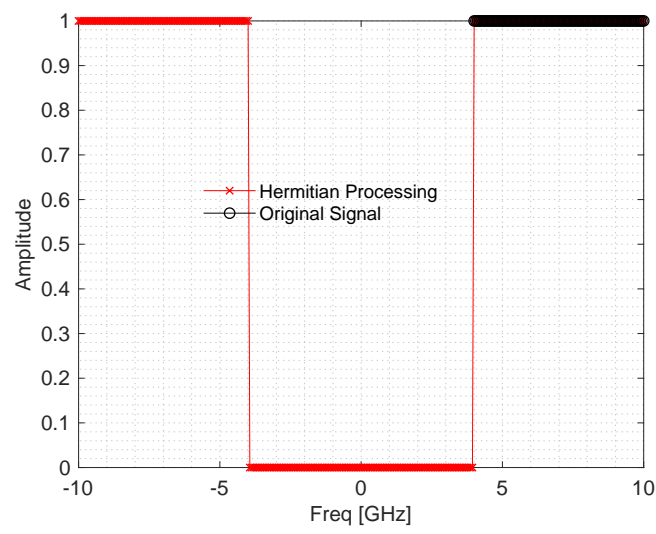

Fig. 4. Original (black) and Hermitian (red) measurements.

inter-sampling distance of the original data. After the IFFT operation, the time pulse extends from 0 and $16.5 \mathrm{~ns}$. Fig. 5 shows the time-domain pulse (limited to $10 \mathrm{~ns}$ ), alongside an oversampled version, in which the number of time samples is increased 8 times by interpolation.

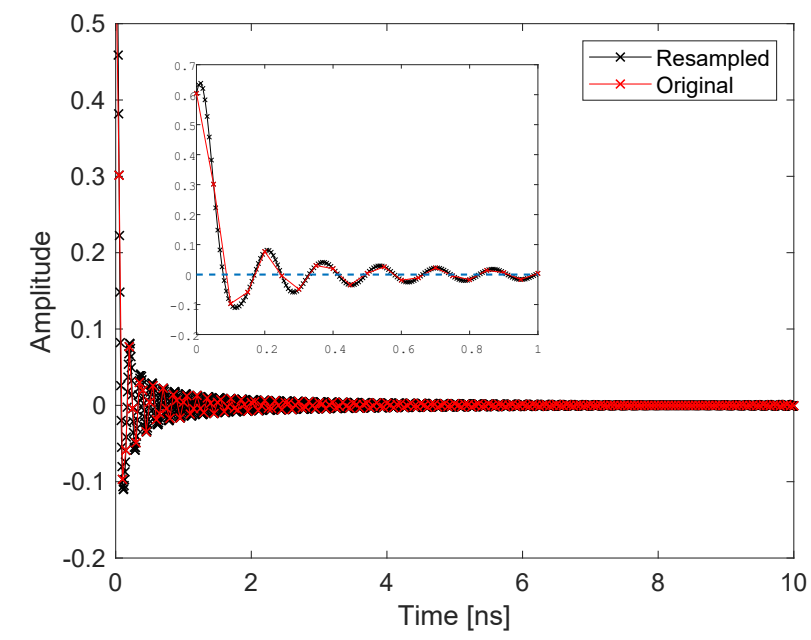

Fig. 5. Transformed time-domain signals (red - original, no oversampling) and (black - 8 times oversampling). In detail the zoomed-in version up to 1 ns.

It can be seen the oscillatory behavior of the time-domain pulse following the sinc shape, that originates after the truncated function rect in the frequency domain, as mentioned before. The next example will show that the phase in the frequency-domain original data conveys distance information 
in applications such as target identification, imaging and TDR.

\section{EXAMPLE II - SHORT CIRCUITS IN TRANSMISSION LINES AND FREE-SPACE}

A short-circuited lossless transmission line of length $l$ and characteristic impedance $Z_{o}$ has its input impedance $Z_{i n}$ written as:

$$
Z_{\text {in }}=j Z_{o} \tan (\beta l)=j Z_{o} \tan \left(\frac{2 \pi f}{c} l\right),
$$

with the variables $f$ and $c$ representing the linear frequency (unit $\mathrm{Hz}$ ) and speed of light, respectively, and $j$ denotes the imaginary unit. The reflection impedance factor $\Gamma$ or the S11 (since there is only one port they are the same) at the input port can be found after:

$$
\Gamma=\frac{Z_{\text {in }}-Z_{o}}{Z_{\text {in }}+Z_{o}}=\frac{j \tan \left(\frac{2 \pi f}{c} l\right)-1}{j \tan \left(\frac{2 \pi f}{c} l\right)+1} .
$$

It can be seen that $\Gamma$ has always a unitary module and only its phase is allowed to vary. In the context of a Smith Chart, the loci of impedance points lie on the chart periphery. This equation (12) is equivalent to the reflection produced on an antenna by $N$ targets, located at distances $x_{i}$ [1]:

$$
\Gamma=\sum_{i=1}^{N} A_{i} e^{-j \frac{2 \pi f}{c} 2 x_{i}}
$$

where the factor 2 multiplying the distance variable accounts for the round-trip length. This equation resembles a DFT (Discrete Fourier Transform) and is the basics behind ISAR (Inverse Synthetic Aperture Radar) [1]. $A_{i}$ stands for the reflection amplitude, which for the case of a PEC (perfect electric conductor) is unitary, meaning that the reflected wave has the same amplitude as the incident wave.

In order to investigate it, two short circuits located at a distance of 0.2 and 0.6 meters were simulated from DC to $10 \mathrm{GHz}$ using a generic circuit simulator and by means of equations (12) and (13) - the latter representing a metallic plane located at the same distances, scattering the energy back to the antenna. All three analyses resulted in the same data for the $\Gamma$ or S11, as shown in Fig. 6 with reduced bandwidth for a better visualization.

After Hermitian Processing, which in this case did not need zero-padding since $f_{\min }=0$, the IFFT is performed and the time-domain pulses are visualized in Fig. 7, after oversampled by a factor of 8 . Two interrupted blue lines were added to show the theoretical delay time (twice the target distance over velocity of light), showing that the scaling implemented by the IFFT in the time axis is consistent.

\section{EXAMPle III - Free SPACE SyStem, ANTENNA AND METALLIC TARGET}

For the purpose of near-field or far-field imaging, antennas are used as probes. Similarly to radars, the imaging system can be called monostatic, where the same antenna is used for either transmission and reception, or bistatic, where there are different antennas for each purpose [19]. Based on the measured
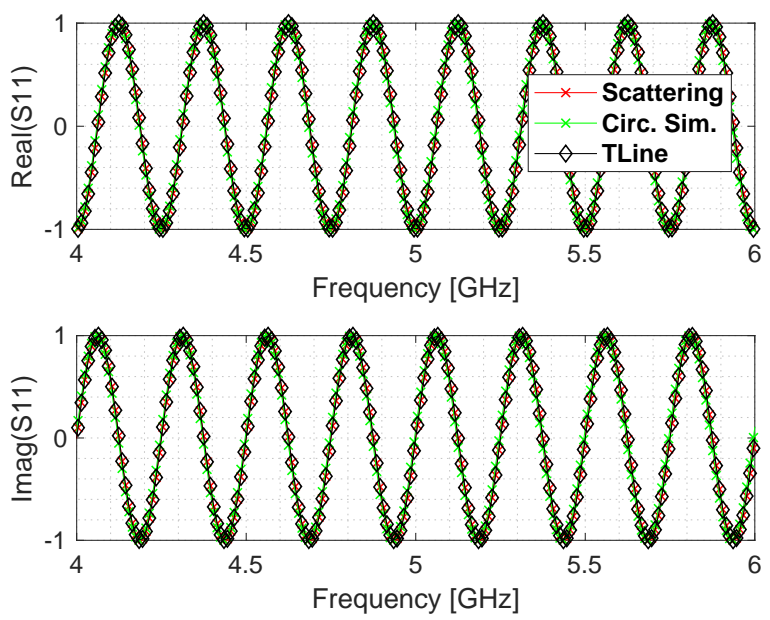

Fig. 6. S11 or $\Gamma$ for a short circuit/target located at distance of $0.6 \mathrm{~m}$. The scattering equation is (13) and Transmission line is (12).

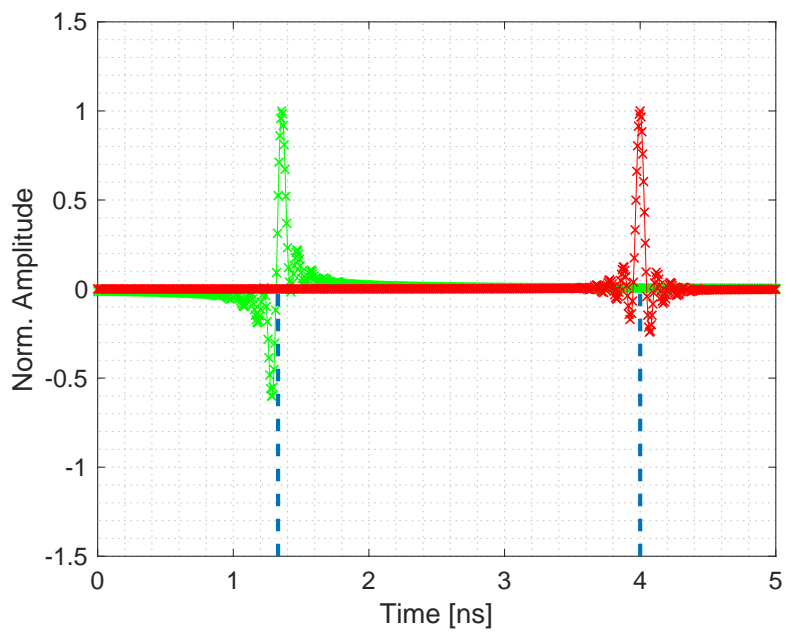

Fig. 7. Time responses corresponding to the $\mathrm{S} 11$ of a short circuit (or metallic target in free space) located at 0.2 (green) and $0.6 \mathrm{~m}$ (red).

antenna response, the target image can be obtained using either inversion or direct processing. Inverse scattering techniques use the available data to back-propagate the information and generate the image. In contrast to direct analysis methods, they are more computationally intensive given the fact the matrix containing the S-parameters from the scene is illconditioned [21]. They usually rely on previous information, simulated or measured, of the environment where the imaging is performed, and disregard multi-path propagation using the Born approximation to simplify the problem [22] [23] [24].

Though phase information is of paramount importance in the process, there are special techniques based only on amplitude information, such as phaseless imaging [25]. For the sake of phase measurement, complex data should be acquired, which means coherent detection is needed. Spectrum analyzers, largely available in laboratories, for instance, only provide amplitude data. Unfortunately, more expensive VNAs or high-frequency oscilloscopes should be used if one needs 
coherent detection.

For the sake of example, a TEM Horn Antenna was simulated alone to extract its isolated characteristics, and radiating against a target, according to Fig. 11. Horns are particularly attractive for imaging purposes given their mechanical sturdiness and high gains, either in far and near-fields [26] [27]. The antenna has inherent broad bandwidth, which is mainly determined by its exponentially flared shape and the balun that connects the coaxial connector to the parallel-plate transmission line. The antenna isolated was simulated and Fig. 8 presents the input reflection in $\mathrm{dB}$, which is the same as the S11 for systems with only one port. Its maximum computed gain is shown in Fig. 9, for endfire direction. It can be seen that the computed $\mathrm{S} 11$ goes below $-10 \mathrm{~dB}$ at frequencies higher than $10 \mathrm{GHz}$. However, $10 \mathrm{GHz}$ was chosen as upper limit in the current analysis in order to make it consistent with UWB legal limits and also to avoid too long simulation times, which are proportional to the maximum frequency.

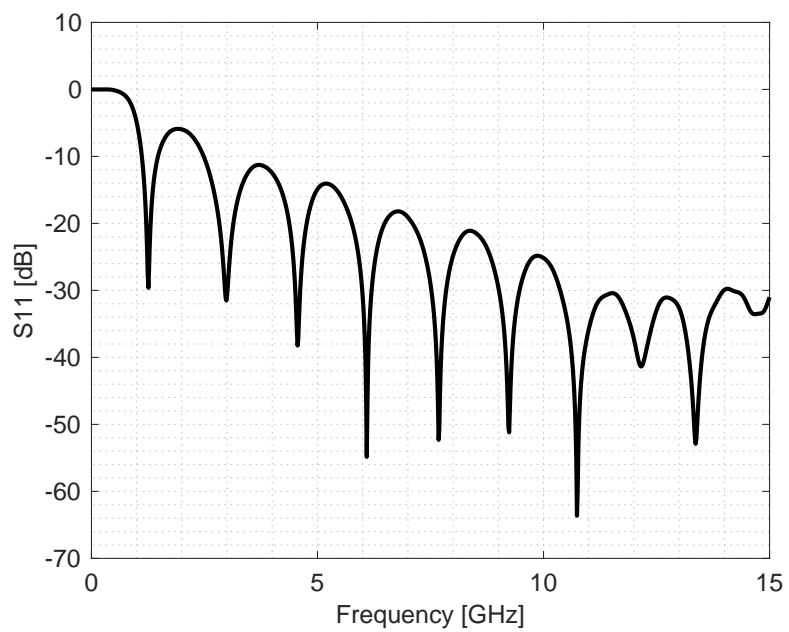

Fig. 8. Computed antenna return loss, S11.

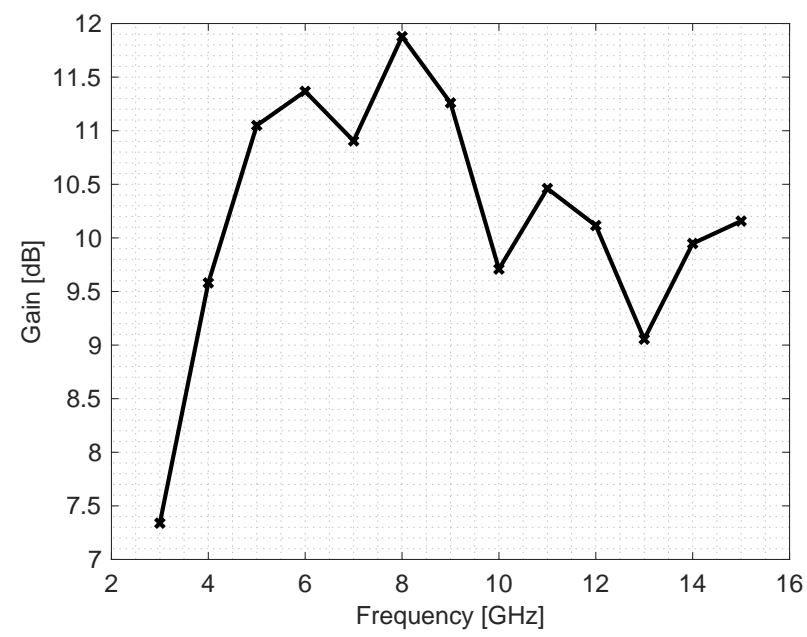

Fig. 9. Computed antenna Gain, in dB.

Fig. 10 shows the gain in a 3D cutplane. It can be seen that for three different frequencies within the analyzed range the direction of maximum radiation is pointing towards the target (endfire direction). It is important that the antenna as a sensor have small side lobes and a null in the back side, conditions that are unfortunately not possible if a high gain is needed [28].
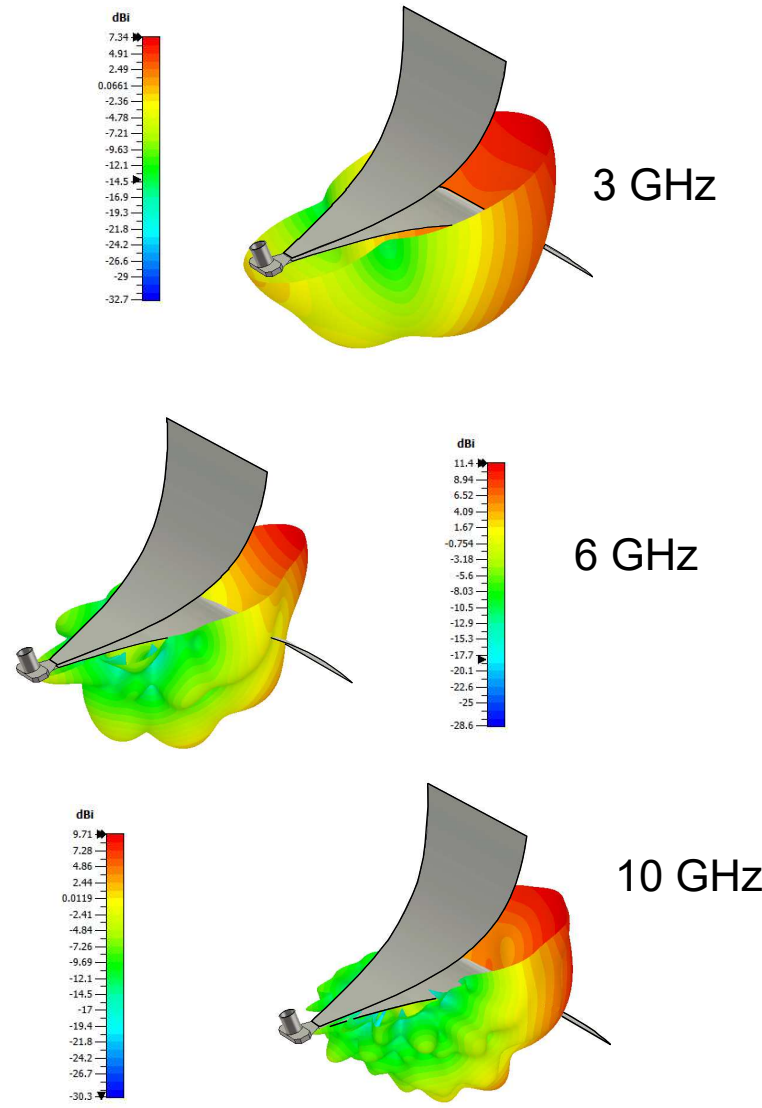

Fig. 10. Computed antenna Gains in 3D, for three different frequencies, in dB.

A metallic obstacle was placed at a distance $(0.1$ and 0.2 $\mathrm{m})$ measured from the antenna aperture middle-point, a square sheet with side length $0.1 \mathrm{~m}$, according to Fig. 11. Define the far-field condition [19] as:

$$
r>\frac{2 D^{2}}{\lambda}
$$

where $r$ is the limit distance where far-field conditions apply and $D$ is the largest antenna dimension. Considering the minimum and maximum frequency $(2.5 \mathrm{and} 10 \mathrm{GHz})$ it results in the limit distance to be respectively 11 and $44 \mathrm{~cm}$, so the target can be supposed to lie in the far-field region. Following the definition of [20], on the other hand:

$$
r>\frac{\lambda}{2 \pi},
$$

it results in $12 \mathrm{~cm}$ and $3 \mathrm{~cm}$ for $2.5 \mathrm{GHz}$ and $10 \mathrm{GHz}$, respectively, thereby placing the target in near-field for the lower frequency range. What really matters is whether the 
antenna input impedance is sensitive enough against the target influence. These modifications in the complex S11 results would encode the target position and geometry.

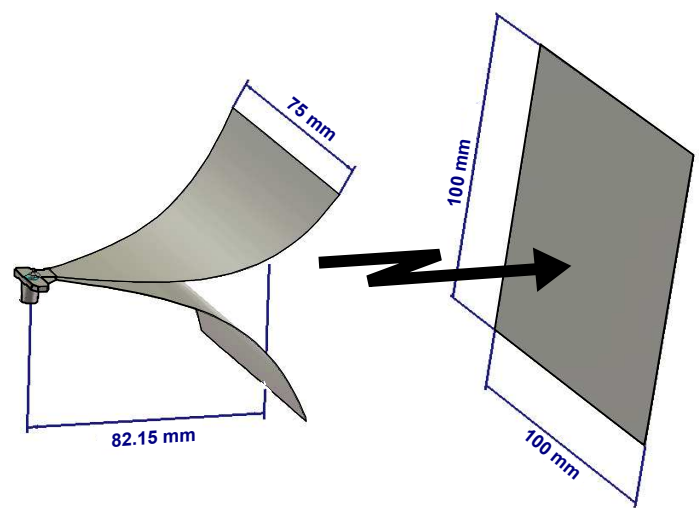

Fig. 11. TEM horn antenna and the target.

Three scenarios were simulated inside CST Microwave Studio - the isolated antenna and the target at the two distances. Fig. 12 contains the simulated scattering parameters, covering the frequency range with 751 equidistant points. The range was chosen as to use the antenna with its S11 parameter operating under $-10 \mathrm{~dB}$. Given the inherent broadband scenario, a FIT (Finite Integration Technique) time-domain solver was used. The S-parameters for each scenario were later exported to an ASCII file.
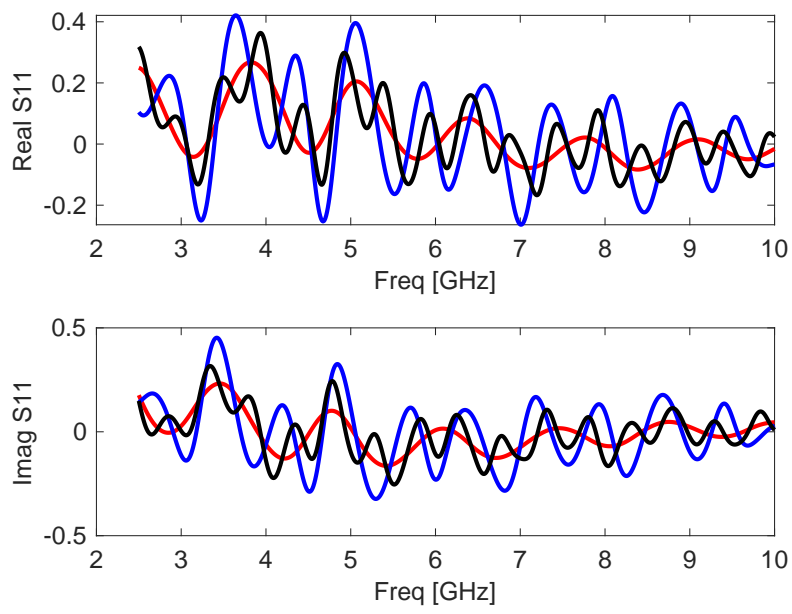

Fig. 12. Real and imaginary input reflection at the antenna input port. Red: Isolated antenna; blue: target at $10 \mathrm{~cm}$ and black: target at $20 \mathrm{~cm}$.

After the Hermitian Processing is performed, the IFFT is computed. In case of an antenna, there are several reflections arising besides the target; all different transitions of the guided wave from the input port to the effective radiation into space generate reflections clearly seen on the time-domain pulse, even if the input impedance of the antenna is well matched within the used frequency range [29]. It is important to stress that the scattered wave amplitude from the target and picked up by the antenna aperture is very low and can be masked by reflections from the antenna itself, even with relatively low
S11 parameters. Therefore, an artifact removal procedure is needed to better isolate the effects of the target alone. Fig. 13 shows the three pulses (isolated antenna and with two targets) zoomed in up to $3 \mathrm{~ns}$. It can be seen that during the pulse onset all the three curves are very similar, accounting for the antenna structure internal reflections. Besides that, as the target is moved away from the antenna aperture, the return pulse amplitude decreases. In a real case, ambient noise, reflections from the environment, and instrument dynamic ranges impose a constraint on the maximum distance in which a target can be identified. Field solvers, on the other hand, are limited only by the numerical and discretization errors, as there is no noise in the virtual model.

Besides mechanical and size constraints, from the viewpoint of electromagnetic performance, the following parameters are important to address which antenna should be used for imaging:

- A larger gain antenna implies a target can be spotted further away;

- A large bandwidth antenna generates faster pulses in the time-domain;

- A constant group delay and a stable center of phase across the used frequency range would help keep the pulse undistorted.

A moving center of phase implies that, in contrast to the previous transmission line example, the origin (distance zero) from where the phase is measured is not constant across the antenna geometry. There is a distortion on the pulse due to the non-constant center of phase [29], which in turn implies a frequency-varying group delay. Routines similar to MIMO (Multiple-Input Multiple-Output) are used for the identification of different targets and, to discard information from multipaths, among other techniques the Singular Value Decomposition (SVD) is employed [30]. For imaging applications, the antenna design can be made to specifically address a constant phase center in order to decrease this source of error [31].

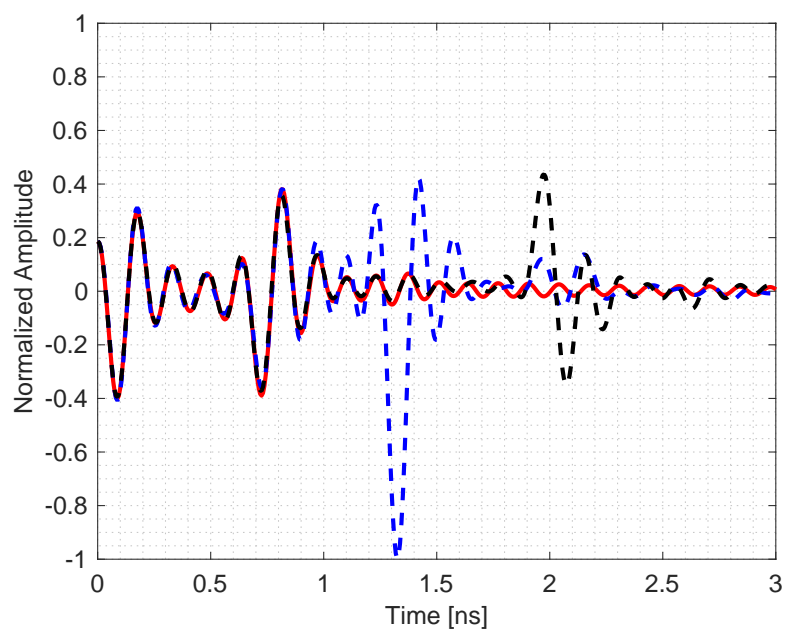

Fig. 13. Pulses in the time domain; blue: target at $10 \mathrm{~cm}$ and black: target at $20 \mathrm{~cm}$. 
In order to eliminate structural reflections from the responses, the pulse shape in the time domain with the antenna only is subtracted from the same pulse with targets. Fig. 14 contains the pulses after subtracting the antenna-only. The time difference between the maxima of both pulses is $0.64 \mathrm{~ns}$ whereas the theoretical value is $0.66 \mathrm{~ns}$; this difference can be accounted for geometrical offsets in the center of phase along the used frequency range. The same reason explains the time of the maxima not being exactly equal to $c / 2 x$, where $x$ is the distance antenna-target. Since the distance in the simulation is considered from the aperture, it does not correspond to the center of phase. Fig. 15 depicts the simulated center of phase variation for the frequency range $3 \mathrm{GHz}$ to $10 \mathrm{GHz}$. It can be seen that the variable position across the bandwidth will impose a different phase error in the time waveshape.

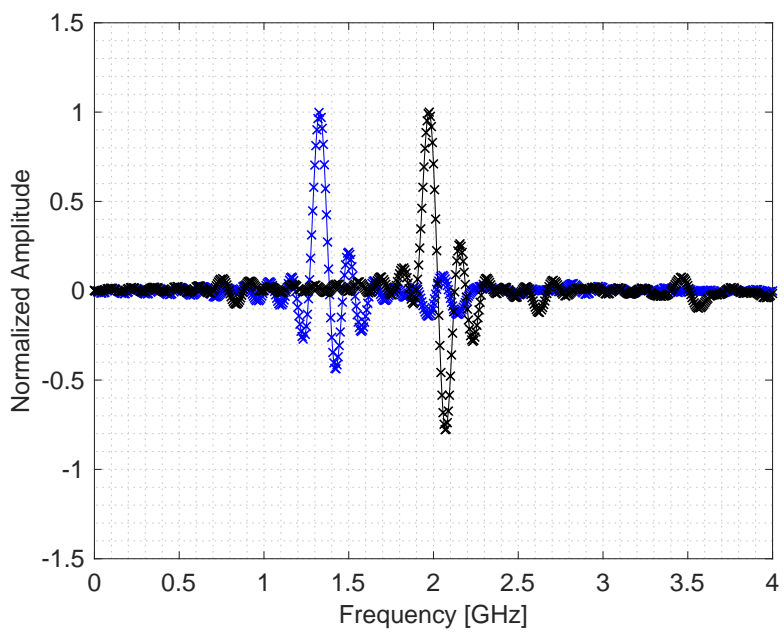

Fig. 14. Pulses in the time domain after subtracting antenna-only; blue: target at $10 \mathrm{~cm}$ and black: target at $20 \mathrm{~cm}$.

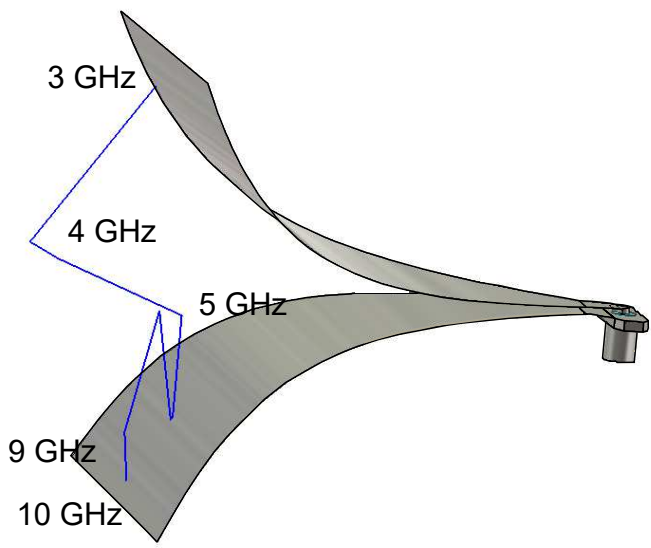

Fig. 15. Simulated loci of phase center for different frequencies.

\section{CONCLUSION}

This paper reported the conversion of S-parameter data to time-domain employing Hermitian Processing, where the original frequency-domain S-parameter represents some physical measurement. We present the necessary steps in the processing with examples that show the physical consistency of the defined rules. Topics regarding antennas for near and far-field imaging are also discussed, as well as trade-offs concerning different parameters, such as antenna characteristics, choice of bandwidth, number of sampling points, thereby providing an overall understanding of the limitations and boundaries given the available hardware. The information presented enables a clearer understanding of the conversion of existing scattering parameter matrices in the frequency domain to time-domain signals

\section{REFERENCES}

[1] C. Özdemir, Inverse Synthetic Aperture Radar Imaging with Matlab ${ }^{\circledR}$ algorithms, Hoboken, NJ, USA: John Wiley \& Sons, 2012.

[2] V. C. Chen and H. Ling, Time-Frequency Transforms for radar imaging and signal analysis. Norwood, MA, USA: Artech House, 2002.

[3] W. Shao and T. McCollough, "Advances in Microwave Near-Field Imaging", IEEE Microw. Mag., vol. 21, no. 5, pp. 94-119, May 2020, doi: 10.1109/MMM.2020.2971375

[4] M. B. Bicer, A. Akdagli and C. Özdemir, "A Matching-Pursuit Based Approach for Detecting and Imaging Breast Cancer Tumor", Prog. Electromagn. Res. $M$, Vol.64, pp. 65-76, 2018, doi: :10.2528/PIERM17101205

[5] H. M. Jol, Ground Penetrating Radar Theory and Applications. Amsterdam, The Netherlands: Elsevier. 2009.

[6] H. C. Rhim and O. Büyüköztürk, "Wideband Microwave Imaging of concrete for nondestructive testing", J. Structural Eng., vol.126, no. 12, pp. 1451-1457, Dec. 2000, doi: 10.1061/(ASCE)07339445(2000)126:12(1451).

[7] M. E. Hines and H. E. Stinehelfer, "Time-Domain Oscillographic Microwave Network Analysis Using Frequency-Domain Data", lEEE Trans. Microw. Theory Techn., Vol. MTT-22, No. 3, pp. 276-282, Mar. 1974, doi: 10.1109/TMTT.1974.1128211.

[8] B. Ulriksson, "Conversion of frequency-domain data to the time domain", Proc. IEEE , Vol. 74, No. 1, pp. 74-77, Jan 1986, doi: 10.1109/PROC.1986.13405.

[9] M. A. Jensen , D. V. Arnold and D. E. Crockett, "System-Level Microwave Design: Radar-Based Laboratory Projects", IEEE Trans. Educ., vol. 43, no. 4, pp. 414-419, Nov. 2000, doi: 10.1109/13.883351.

[10] G. H. Weber, H. L. Moura, G. Dutra, U. J. Dreyer, R. J. Daciuk, J. C. C. da Silva, C. Martelli, D. R. Pipa and M. J. da Silva, "Sparse Inverse Chirp-Z Transform of S-Parameter Measurements for Time Domain Analysis of Transmission Line", in XXXV Simpósio Brasileiro de Telecomunicações e Processamento de Sinais, SBrT, Sep. 2017, pp. 697. 701, doi:10.14209/sbrt.2017.227.

[11] K. M. Chew, R. Sudirman, Y. H. How and C. Y. Yong, "Microwave Signal Spatial Domain Transformation using Signal Processing and Image Reconstruction Method", in 2013 1st Int. Conf. Artif. Intell. Model. Simul., Dec 2013, pp. 98-103, doi: 10.1109/AIMS.2013.23.

[12] K. M. Chew, R. Sudirman, N. H. Mahmood, N. Seman and C. Y. Yong, "Human Brain Microwave Imaging Signal Processing: Frequency Domain (S-Parameters) to Time Domain Conversion", Eng., Vol. 5 No. 5B, pp. 31-36, May 2013, doi: 10.4236/eng.2013.55B007 .

[13] K. M. Chew, R. Sudirman, N. Seman and C. Y. Yong, "Reflection Coefficient Detection of Simulation Models for Microwave Imaging Simulation System", Biomed. Mater. Eng., Vol. 24, no. 1, 2014, pp. 1-7, doi: 10.3233/BME-130800.

[14] S. Pisa, E. Piuzzi, E. Pitella, P. D’Atanasio, A. Zambotti, G. Sacco, "Comparison Between Delay and Sum and Range Migration Algorithms for Image Reconstruction in Through-the-Wall Radar Imaging Systems", IEEE J. Electromagn., RF, Microw. Med. Biol., vol. 2, no. 4, pp. 270-276, Dec. 2018, doi: 10.1109/JERM.2018.2878070.

[15] S. Tantong, B. Camps-Raga, P. Kirawanich and N. E. Islam, "Improved Method for Embedded Object Detection and Reconstruction Through Near-Field Pulse Imaging Techniques ",in 2007 IEEE 34th Int. Conf. Plasma Sci., Jun. 2007, pp. 630-634, doi: 10.1109/PPPS.2007.4345758

[16] I. Oppermann, M. Hämäläinen and J. Iinatti, UWB Theory and Applications. West Sussex, UK: John Wiley and Sons, 2004.

[17] S. Sercu, C. Kocuba, J. Nadolny, "Causality Demystified", in DesignCon 2015, Jan. 2015.

[18] R. N. Bracewell, The Fourier Transform and its applications, 3rd Edition. Boston, MA, USA: McGraw Hill, 2000. 
[19] C. A. Balanis, Antenna Theory: Analysis and Design, 2nd Edition. New York, NY, USA: John Wiley \& Sons, 1997.

[20] H. W. Ott, Electromagnetic Compatibility Engineering. Hoboken, NJ, USA: Wiley, 2009.

[21] J. M. Felicio, J. M. Bioucas-Dias, J. R. Costa and C. A. Fernandes, "Microwave Breast Imaging Using a Dry Setup", IEEE Trans. Comput. Imag., vol. 6, pp. 167-180, Jul. 2019, doi: 10.1109/TCI.2019.2931079.

[22] J. Sachs, S. Ley, T. Just, S. Chamaani and M. Helbig, "Differential Ultra-Wideband Microwave Imaging: Principle Application Challenges", Sensors, vol. 18, no. 7, Jul. 2018, doi: /10.3390/s18072136.

[23] N. Bayat and P. Mojabi, "On an Antenna Design for 2D Scalar NearField Microwave Tomography", ACES J., vol. 30, no. 6, pp. 589-598, June 2015.

[24] M. E. Bialkowski, Y. Wang, A. A. Bakar and W. C. Khor, "Microwave imaging using ultra wideband frequency-domain data", $M i$ crow. Opt. Technol. Lett., vol. 54, No. 1, pp. 13-18, Jan. 2012, doi: 10.1002/mop.26465.

[25] O. Yurduseven, T. Fromenteze, D. L. Marks, J. N. Gollub and D. R. Smith, "Frequency-Diverse Computational Microwave Phaseless Imaging", IEEE Antennas Wireless Propag. Lett., vol. 16, pp. 2808-2811, Sep. 2017, doi: 10.1109/LAWP.2017.2748139.

[26] R. A. Amineh, M. Ravan, A. Trehan and N. Nikolova, "Near-Field Microwave Imaging Based on Aperture Raster Scanning with TEM Horn Antennas", IEEE Trans. Antennas Propag., vol. 59, no. 3, pp. 928-940, March 2011, doi: 10.1109/TAP.2010.2103009.

[27] E. C. V. Boas, J. A. P. Ribeiro, K. L. M. Costa, J. A. J. Ribeiro, A. M. Alberti and A. C. Sodre Jr, "Design and Characterization of UltraWideband Horn Antenna in Quasi-TEM Mode", J. Commun. Inf. Syst., vol. 38 , no. 1, pp. 339-346, Nov. 2018, doi: 10.14209/jcis.2018.34

[28] S. Gregson, J. McCormick and C. Parini, Principles of Planar Near Field Antenna Measurements. London, UK: IET Institute of Engineering and Technology, 2007.

[29] J. M. Felicio, J. M. Bioucas-Dias, J. R. Costa and C. A. Fernandes, "Antenna Design and Near-Field Characterization for Medical Microwave Imaging Applications", IEEE Trans. Antennas Propag., vol. 67, no. 7, pp. 4811-4824, Jul. 2019, doi: 10.1109/TAP.2019.2905742.

[30] G. G. Rayleigh and J. M. Cioffi, "Spatio-temporal coding for wireless communications", in IEEE Global Telecommun. Conf., Nov. 2006, pp. 1809-1814, doi: 10.1109/GLOCOM.1996.591950

[31] A. Hatami, A. S. Arezomand and F. B. Zarrabi, "Phase center controlling in Vivaldi antenna: review and development of the story", J. Comput. Electron., vol. 19, no.4, pp. pp. 736-749, Feb. 2020, doi: 10.1007/s10825020-01463-z.

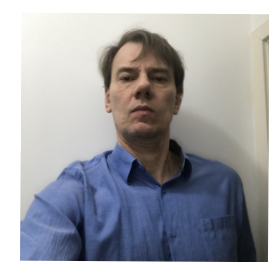

Marcelo B. Perotoni Elec. Eng. (1995, UFRGS, Porto Alegre, Brazil), MsC (2001) and PhD (2005) in Electrical Engineering from USP (Sao Paulo, Brazil). Interests in RF and EMC. He is currently a professor at UFABC.

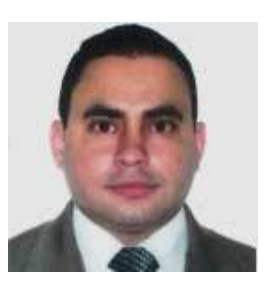

Kenedy M. G. dos Santos Elec. Eng. (2006, PUCMG, Belo Horizonte, Brazil), M.Sc. in Electrical Engineering (2011,UFMG, Belo Horizonte, Brazil) and PhD. in Electrical Engineering (2018, UFBA, Salvador, Brazil). Currently is professor at the IFBA and has experience in Electrical Engineering with emphasis on EMC, Microwave and Antennas.

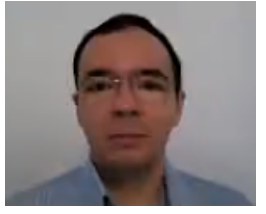

Claudio J. Bordin Jr. Claudio J. Bordin Jr. received the B.S., M.S., and Ph.D. degrees, all in Electrical Engineering, from Escola Politécnica, Universidade de São Paulo, Brazil, in 2000, 2002, and 2006, respectively. He is currently an Associate Professor at Universidade Federal do ABC, Brazil. His research interests lie in the area of Signal Processing and Applied Statistics. 